Itinéraires Itinéraires

Littérature, textes, cultures

2013-2 | 2014

Sade et les femmes

\title{
Entre le crime et la sensibilité
}

Les paradoxes du personnage de Clairwil

\section{Clara Carnicero de Castro}

\section{(2) OpenEdition \\ Journals}

Édition électronique

URL : http://journals.openedition.org/itineraires/652

DOI : 10.4000/itineraires.652

ISSN : 2427-920X

Éditeur

Pléiade

\section{Édition imprimée}

Date de publication : 1 mars 2014

Pagination : 33-44

ISBN : 978-2-343-02712-8

ISSN : $2100-1340$

Référence électronique

Clara Carnicero de Castro, "Entre le crime et la sensibilité », Itinéraires [En ligne], 2013-2 | 2014, mis en ligne le 01 novembre 2013, consulté le 21 avril 2019. URL : http://journals.openedition.org/ itineraires/652 ; DOI : 10.4000/itineraires.652

\section{(c) (i) (9)}

Itinéraires est mis à disposition selon les termes de la licence Creative Commons Attribution - Pas d'Utilisation Commerciale - Pas de Modification 4.0 International. 


\section{Entre le crime et la sensibilité}

\section{Les paradoxes du personnage de Clairwil}

\section{Résumé}

Héroïne complexe, Clairwil s'impose avec ses contradictions. Comme la marquise de Merteuil, elle aime venger son sexe de la suprématie masculine et énonce un système d'apathie complexe. Pourtant, son féminisme est chancelant, car il suppose la faiblesse de la femme, qui prétend offenser la nature par le triomphe de la faiblesse. Quant au stoïcisme, l'institutrice libertine exige que Juliette ne se livre au crime que de sangfroid et assure : «Chez moi tout est aux ordres de ma tête ». Mais l'heureuse apathie et le féminisme militant sont facilement dissipés. Le putanisme fanatique, l'enthousiasme blasphématoire ou le fantasme d'un crime pur et perpétuel remplacent la froide raison. Sa vie finit par le refus d'un crime singulier : tuer sa chère écolière. L'apprentie, en revanche, ne balance pas, elle empoisonne l'enseignante flegmatiquement. À l'instar de Merteuil, le destin de Clairwil est tragique : à force de surestimer l'impassibilité de la tête, elle sous-estime l'impétuosité fascinante de Juliette.

Mots clés : Sade, Clairwil, crime, sensibilité, apathie

\section{Abstract}

Clairwil is a complex heroine characterized by contradictions. As the Marquise de Merteuil she enjoys avenging her sex over male supremacy and articulates a sophisticated system of apathy. However her feminism is faltering, for it presupposes the weakness of woman who hopes to offend Nature through the triumph over strength. As for stoicism, the libertine teacher demands that Juliette only commit crime in cold blood and maintains: the mind must control everything. Yet, fortunate apathy and militant feminism are easily dispelled. Fanatical putanisme, enthusiastic blasphemy and the fantasy of pure and perpetual crime replace cold reason. Her life ends by refusing an uncommon crime: killing her beloved pupil. The apprentice, on the contrary does not hesitate, she poisons her mistress phlegmatically. Like Merteuil Clairwil's destiny is tragic: by overestimating the impassivity of reason, she underestimates Juliette's fascinating impetuousness.

Keywords: Sade, Clairwil, crime, sensitivity, apathy 
L'Histoire de Juliette est le récit d'une éducation permanente. Dans la deuxième partie du roman, Noirceuil tient un discours sur l'hypocrisie pour enseigner à la protagoniste le besoin de dissimuler ${ }^{1}$. Comme exemple de comportement, il cite la marquise de Brinvilliers (p. 408), célèbre empoisonneuse. Juliette est au courant des aventures de la meurtrière $\mathrm{du} \mathrm{XVII}^{\mathrm{e}}$ siècle et prétend en être digne, mais préfère comme modèle une femme plus proche d'elle, plus âgée et expérimentée - une contemporaine. L'amie désirée doit avoir une sorte d'empire, mais permettre tous les écarts sans jalousie. Il est question d'une femme « sans religion comme sans principes; sans mœurs comme sans vertu, infiniment de chaleur dans l'esprit, et le cœur à la glace » (p. 409). Heureusement, le libertin connaît la personne que l'apprentie réclame : " c'est une veuve âgée de trente ans, d'une beauté rare, scélérate au dernier degré » (ibid.). L'arrangement tombe à merveille, car Noirceuil prend une nouvelle épouse et ne peut plus accompagner son élève avec la même ardeur. Mme de Clairwil le remplacera dans l'éducation de Juliette. En marge des instituteurs mâles, la jeune courtisane cherche toujours la compagnie savante et la luxure inventive d'une femme. Le " principe d'une complicité ${ }^{2}$ » féminine suit la gradation du roman ${ }^{3}$ et, par conséquent, la formation de la protagoniste : la Delbène transmet d'abord à la novice l'essentiel du savoir philosophique et érotique; puis, c'est à Clairwil de révéler les astuces sociales et criminelles; enfin, la Durand transmet des connaissances techniques et magiques. Si les précepteurs évoquent fatalement le despotisme des passions en échange de leurs privilèges financiers et politiques, les préceptrices incarnent plutôt l'entente saphique. C'est peut-être la raison pour laquelle l'affection des libertins se limite à l'amitié, tandis que celle des libertines progresse jusqu'à l'adoration. Clairwil, comme nous aimerions le montrer, se situe à mi-chemin de cette progression.

\section{Les portraits d'une scélérate}

La transition entre les différents modèles de libertinage féminin se traduit toujours par un portrait. Celui de Clairwil se présente comme l'un des plus ingénieux du roman. Dès leur premier rendez-vous, Juliette dessine le tableau de sa nouvelle institutrice. Les traits corporels de la débauchée accentuent son caractère autoritaire, explicité quelques lignes plus loin :

1. Sade, Histoire de Juliette, ou les Prospérités du vice, dans Euvres, éd. Michel Delon, Paris, Gallimard, coll. «Bibliothèque de la Pléiade », t. III, 1998, p. 407-408. Les références à cette œuvre seront désormais indiquées dans le texte entre parenthèses.

2. Michel Delon, « Notice de l'Histoire de Juliette », dans Sade, Euvres, op. cit., t. III, p. 1365.

3. Sur la gradation chez Sade, voir Michel Delon, " Notes de l'Histoire de Juliette », op. cit., p. 1423 et 1502. Voir aussi Le Savoir-Vivre libertin, Paris, Hachette, 2000, p. 93. 
Elle était grande, faite à peindre; le feu de ses regards était tel, qu'il devenait impossible de la fixer; mais ses yeux, grands et très noirs, en imposaient plus qu'ils ne plaisaient, et en général, l'ensemble de cette femme était plus majestueux qu'agréable; sa bouche, un peu ronde, était fraîche et voluptueuse, ses cheveux, noirs comme du jais, descendaient au bas de ses cuisses; son nez singulièrement bien coupé, son front noble et majestueux, sa gorge moulée, la plus belle peau, quoique brune [...]. Cependant, soit que je fusse plus jeune, soit que ma physionomie eût en grâce ce que celle-ci avait en noblesse, je plaisais davantage à tous les hommes : Mme de Clairwil surprenait; je me contentais d'enchaîner : elle contraignait l'hommage des hommes, et moi je le dérobais. (p. 418)

Ce portrait physique est suivi par une description morale. Clairwil possède de prime abord les vertus de tout grand libertin : un esprit très élevé, une instruction exceptionnelle et une horreur des préjugés, "déracinés par elle dès l'enfance » (ibid.). À cela s'ajoutent la maîtrise de la chimie, la physique, la philosophie, l'histoire, la géographie, l'anglais et l'italien. En complément, elle dispose de talents artistiques et mondains : le dessin, la musique, la comédie, la danse et l'écriture en vers ou en prose. Son seul défaut est un « orgueil insupportable » (ibid.), effet des « extravagances du bel-esprit » (ibid.) poussées trop loin. Assez riche, elle possède un hôtel à Paris, une maison à la campagne, peu d'amis et beaucoup d'adulateurs. Veuve sans enfant, elle jouit d'une santé de fer et manifeste une «petite dureté » (ibid.), c'est-à-dire une complète insensibilité : pas de larmes, pas de bienfaisance.

Comme le portrait du ministre de Saint-Fond, celui de la friponne s'inscrit dans la traditionnelle critique de la vanité sociale chez les moralistes ${ }^{4}$. À la fin du chapitre "Des biens de fortune ", dans Les Caractères ${ }^{5}$, La Bruyère confronte, physiquement et moralement, la vanité du riche Giton à la discrétion du pauvre Phédon. Le double portrait exacerbe le contraste : teint frais, visage plein et estomac haut chez Giton; teint échauffé, visage maigre et corps sec chez Phédon. L'un parle haut, l'autre articule mal. Recourant à une méthode similaire, Sade établit un rapport direct entre corps et comportement. Juliette a la peau blanche, elle est gracieuse et délicate. Clairwil a la peau brune, le regard de feu, elle est grande et majestueuse. La mémorialiste, encore jeune, a la modestie et la complaisance que sa position exige. Elle n'est pas issue d'une ancienne noblesse - son père était banquier - et ses connaissances restent modestes; mais grâce à sa jeunesse et à sa docilité, elle plaît naturellement. La veuve est noble, plus âgée et occupe le poste de maîtresse, place qui présuppose l'autorité

4. Voir Michel Delon, " "La décharge de Saint-Fond était brillante". Éloge et critique, chez Sade, de l'ostentation sociale ", dans Anne Chamayou (dir.), La Littérature et le Brillant : en hommage à Pierre Malandain, Arras, Artois Presses Université, 2002, p. 206. 5. Jean de la Bruyère, Les Caractères ou les Mours de ce siècle [1688], dans Euvres complètes, Paris, Gallimard, coll. «Bibliothèque de la Pléiade », 1988, p. 200-201. 
et le savoir. Même privée des charmes de la jeunesse, elle met en valeur les prérogatives de sa classe, ses dons intellectuels et ses ressources financières. L'arrogance et le mépris renforcent son empire. Comme chez Saint-Fond, le libertinage de Clairwil est une « liberté élitiste, un privilège de disposer en maitre de son propre corps et du corps des autres, une assurance d'impunité ${ }^{6} \gg$.

Clairwil n'est-elle dès lors qu'un personnage type? Chaque libertin semble plutôt « unique en son genre » (p. 1261) et révèle une épaisseur moins psychologique qu'érotique ou discursive. Aux deux tableaux de l'institutrice ébauchés par Juliette, s'ajoute un autoportrait qui élargit son caractère :

Mon âme est impassible [...]; je défie aucun sentiment de l'atteindre, excepté celui du plaisir. Je suis maîtresse des affections de cette âme... de ses désirs... de ses mouvements; chez moi tout est aux ordres de ma tête; et c'est ce qu'il y a de pis [...] car cette tête est bien détestable; mais je ne m'en plains pas, j'aime mes vices, j'abhorre la vertu; je suis l'ennemie jurée de toutes les religions, de tous les dieux, je ne crains ni les maux de la vie, ni les suites de la mort. (p. 419)

D'abord, Clairwil privilégie sur la sensibilité morale, les sensations physiques. Elle assujettit ensuite le plaisir des sens au contrôle de la raison et annonce « la prééminence de l'intelligence sur les mécanismes physio$\operatorname{logiques}^{7} \gg$. Quoique l'ancienne hiérarchie, qui soumet le corps à l'esprit, soit radicalement contestée chez $\mathrm{Sade}^{8}$, la dimension qu'elle revêt dans ce passage ne saurait être anodine. Cette exception se justifie par la construction du personnage, dont les paradoxes enrichissent le caractère. Chez la veuve, la primauté de la tête est plus rhétorique que pratique. Affermie par une théorie complexe de l'apathie, l'héroïne souligne son sang-froid. Cependant, lorsqu'elle cherche à s'imposer aux autres, elle atténue les tournures délicates ou enthousiastes de sa personnalité.

Sade a-t-il puisé le modèle de sa libertine chez Laclos? Annie Le Brun s'étonne que la critique n'ait pas vu dans la marquise de Merteuil une aînée de Juliette ${ }^{9}$. Elle admet néanmoins que cette lacune critique puisse être fondée, la veuve de Liaisons dangereuses se présentant comme « le fascinant personnage-idée que Sade semble justement se refuser de concevoir pour donner vie à Juliette ${ }^{10} »$. Une ressemblance plus fine s'établit ainsi entre

6. Michel Delon, «"La décharge de Saint-Fond" », op. cit., p. 207.

7. Arlette André, «Sade et l'éthique de l'apathie », dans Mélanges littéraires François Germain, Dijon, Faculté de lettres et philosophie de Dijon, 1979, p. 95-104, p. 101.

8. La physiologie joue en effet un rôle primordial, voir ibid., p. 100-101.

9. Voir Jean Biou, « Deux œuvres complémentaires : Les Liaisons dangereuses et Juliette », dans Le Marquis de Sade, Paris, Armand Colin, 1968, p. 103 et André Delmas, À la recherche des «Liaisons dangereuses », Paris, Mercure de France, 1964, p. 49.

10. Annie Le Brun, Sade, aller et détours, Paris, Plon, 1989, p. 88. 
Merteuil et Clairwil. Les deux femmes sont des manipulatrices de métier et partagent un « élitisme hautain et méprisant ${ }^{11}$ ». Représentantes d'un « libertinage au féminin ${ }^{12} »$, elles repoussent la soumission à laquelle la société les a contraintes et décident de venger leur sexe de la suprématie masculine. Elles se prévalent finalement d'être des femmes de têtes et condamnent les faiblesses sentimentales, professant leur théorie sur l'apathie.

\section{L'heureuse apathie}

Chez Clairwil, ce libertinage au féminin se traduit par une attirance sexuelle pour les femmes et par une répulsion criminelle pour les hommes. En voyant l'apprentie émerveillée par son expérience saphique, l'institutrice révèle qu'elle adore les femmes et méprise les hommes au point de leur réserver sa cruauté (p. 421). Fière de son sexe, elle veut immoler tous ceux dont les regards peuvent l'avilir (ibid.). Juliette doute du sang-froid de son enseignante et cherche à s'assurer qu'elle pratique effectivement ce qu'elle prêche. Clairwil ne transige pas : «Mon âme est dure, et je suis loin de croire la sensibilité préférable à l'heureuse apathie dont je jouis » (ibid.). La réponse exige des développements qui débouchent sur une dissertation sur la sensibilité.

Selon la dépravée, la sensibilité est physique et ne dépend que de la conformité des organes, de la délicatesse des sens et surtout de la nature du fluide électrique qui circule dans les concavités des nerfs (p. 422). Le rapport des objets extérieurs produit toujours un genre d'inflammation sur les particules du fluide nerveux. Cette sensibilité peut avoir deux effets opposés : si l'embrasement des particules est faible et lent, il inspire la vertu et on ressent une sensation plus inerte; mais si l'agitation est forte et rapide, elle incite le vice et on éprouve une secousse plus énergique. En somme, « la sensibilité n'est que mécanique, [...] c'est d'elle d'où tout naît, et [...] c'est elle qui nous conduit à tout » (ibid.). Toutefois, il faut émousser radicalement la sensibilité chez une jeune personne, car son excès la porterait aux commotions passives - la pitié, la bienfaisance, l'amour - ou aux commotions énergiques, mais irrationnelles - le crime mal conçu. Or, il semble que le raisonnement de Clairwil aboutisse à un nouveau paradoxe : comment le jeune individu goûtera-t-il les plaisirs des sens s'il a été programmé pour ne rien sentir?

La question est complexe et requiert plusieurs interprétations. Si l'on en croit l'analyse de Michel Delon ${ }^{13}$, la dissertation de Clairwil se développe par renversement en deux temps. Le premier est amoral : vice et vertu sont identifiés à une pulsion, la sensibilité. Dans ce cas-là, émousser

11. Michel Delon, Le Savoir-Vivre libertin, op. cit., p. 286.

12. Nous empruntons cette expression à Michel Delon (ibid., p. 281).

13. Michel Delon, L'Idée d'énergie au tournant des Lumières (1770-1820), Paris, PUF, 1988, p. 408. 
la sensibilité signifie assurer la tranquillité sociale de l'élève, le préserver de l'infortune. L'apathie, recommandée pour que le jeune ne soit pas puni, serait donc l'antonyme de l'énergie. Le deuxième temps est immoral : les vices énergiques s'opposent aux vertus inertes. Le flegme serait synonyme d'énergie, le ressort d'un libertinage criminel. Ainsi, les termes « sensibilité » et " passion » sont ambivalents : ils ont une connotation négative lorsqu'ils révèlent une identification à autrui, mais positive s'ils sont rapportés à la recherche égoïste du plaisir.

Le but de l'apathie serait donc d'émousser la sensibilité morale, pour exalter ensuite la sensibilité physique. L'apprenti doit en effet se prémunir contre les dangers de la commisération, de l'effervescence et du sentimentalisme. Les idées de Clairwil s'inscrivent dans la critique de la morale du sentiment ${ }^{14}$ et de l'enthousiasme irrationnel ${ }^{15}$. En conséquence, le stoïcisme réclamé par le personnage ne s'oppose pas forcément aux voluptés : «Malgré la référence stoïcienne, l'apathie est moins [...] un détachement des passions que la prise en charge par la froide raison de leur pire délire. [...] Les principes rationnels servent à tétaniser la passion, à la fixer sous sa forme paroxystique, sans craindre lassitude ni faiblesse ${ }^{16} \gg$. Persuadée de l'efficacité de son système, Clairwil termine sa dissertation en attendant de voir son écolière poursuivre le même chemin : «Voilà les principes qui m'ont amenée à cette tranquillité... à ce repos des passions... à ce stoïcisme qui me permet maintenant de tout faire, et de tout soutenir sans émotion; presse-toi donc de t'initier à ces mystères » (p. 426).

Les problèmes ne se font pas attendre. Juliette a saisi le besoin de l'apathie, elle sait pourtant que la prudence glace les plaisirs. Sa « nature enthousiaste ${ }^{17} »$, impétueuse et parfois même sensible ne s'accorde pas avec les systèmes fermés de l'institutrice. Au retour d'une orgie à la Société des Amis du Crime, Clairwil blâme l'effervescence de l'apprentie et son manque de flegme :

Ta conscience n'est pas encore où je la voudrais; ce que j'exige est qu'elle devienne tellement tordue qu'elle ne puisse jamais se redresser; il faudrait employer mes moyens, pour en venir là; je te les indiquerai si tu veux, mais je crains que tu n'aies pas la force de les mettre en usage. (p. 581, italique de l'auteur)

La critique tombe mal. La jeune femme s'intéresse beaucoup aux moyens de ses tuteurs, mais elle a une façon particulière de les employer. Les principes qu'elle apprend sont subvertis.

14. Voir Jacques Domenech, L'Éthique des Lumières : les fondements de la morale dans la philosophie française du XVIII siècle, Paris, Vrin, 1989, p. 59.

15. Voir Michel Delon, L'Idée d'énergie, op. cit., p. 367-369.

16. Ibid., p. 408.

17. Voir Catherine Cusset, «Sade, Machiavel et Néron : de la théorie politique à l'imaginaire libertin », Dix-huitième siècle, nº 22, 1990, p. 406. 


\section{Un féminisme militant?}

Les divergences entre les deux libertines ne sont pas une nouveauté. Si elles sont enchantées l'une de l'autre, trouvant une ressemblance qui resserre leur liaison, une tension s'annonce dès leurs premières aventures. À la fin de son noviciat à la Société des Amis du Crime, Juliette reçoit la permission d'entrer dans les sérails. Clairwil la mène à celui de filles, mais l'écolière insiste pour rendre visite aux garçons. Contre son gré, l'enseignante satisfait le désir de l'apprentie, à la condition qu'aucun membre viril ne jouisse de son corps. La jeune femme accepte, mais le délire est si grand que l'arrangement établi se trouble, laissant les deux libertines pleines de « foutre » (p. 577) sur leur sofa. L'excès est tel que l'institutrice fait venir des fustigateurs. Les «fesses » (ibid.) en feu, elle décide de passer aux scènes plus sérieuses :

As-tu remarqué ce joli garçon de dix-huit ans, qui nous baisait avec tant d'ardeur... [...] Faisons-le passer dans la salle des tourments, nous l'égorgerons.

- Friponne, tu ne m'as point fait la même proposition dans le sérail des femmes?

— Non, j'aime mieux massacrer des hommes, je te l'ai dit, j'aime à venger mon sexe; et s'il est vrai que celui-là ait une supériorité sur le nôtre, l'imaginaire offense à la nature, n'est-elle pas plus grave en l'immolant?

- On te croirait désolée de ce que cette offense est nulle.

- Tu me juges bien, je suis au désespoir de ne trouver jamais que le préjugé, au lieu du crime que je désire et que je ne rencontre nulle part. (p. 578)

S'appuyant sur la prétendue infériorité des femmes, Clairwil trace l'esquisse d'un étrange "féminisme ». Sade n'emploie évidemment jamais ce mot, anachronique, mais comme l'a observé Michel Delon, mis en relation avec la « lucidité féministe $e^{18}$ » de Merteuil, « cet anachronisme lexical s'impose en effet ${ }^{19} »$. D'abord, par les termes revendicatifs dont les deux scélérates se servent : on y retrouve l'écho du débat sur le statut social et politique de la femme à la fin du XVIII e siècle; puis par la place essentielle qu'elles jouent dans l'intrigue ${ }^{20}$. Chez Clairwil, cependant, la revendication est moins nette que chez Merteuil. Selon la première, le massacre des hommes est un puissant écart parce qu'il offense la nature en violant la loi du plus fort (p. 646). Le triomphe de la force n'entraîne jamais de rupture : il s'agit d'un effet naturel. Le triomphe de la faiblesse, en revanche, produit une transgression importante qui déclenche les plaisirs intenses : « Qu'estce que le triomphe de la force sur la faiblesse? Ce qui est tout simple, peut-il amuser? Mais qu'elles sont flatteuses, qu'elles sont douces les victoires remportées par la faiblesse sur la supériorité » (ibid.). Juliette a vite perçu

18. Michel Delon, Le Savoir-Vivre libertin, op. cit., p. 286 (italique de l'auteur).

19. Ibid.

20. Ibid., p. 294-295. 
le préjugé de son amie et affirme indirectement que la transgression désirée est une grande illusion. Même s'il s'agit au fond d'une fantaisie, Clairwil se justifie par l'impossibilité de commettre un vrai crime, compte tenu du déterminisme de la nature.

Dans son rapport avec les hommes, la scélérate expose « le paradoxe de la perversion qui doit poser la loi pour mieux la transgresser ${ }^{21} »$. Si la nature a besoin du vice pour maintenir son équilibre, le libertin n'opère aucune transgression en faisant le mal, il ne sert que la conservation de l'univers. De ce point de vue, le criminel joue un rôle bénéfique : il n'y a pas de crime pour une nature marâtre, tout lui est utile, car tout a été préalablement déterminé. Néanmoins, la volupté libertine exige la transgression et conteste tout ordre prédéfini. Le scélérat est fatalement enfermé dans cette impasse : il abhorre et attaque toute loi, mais il est obligé de la rétablir pour jouir du plaisir de la transgression.

\section{L'enthousiasme blasphématoire}

Ce paradoxe se montre sous plusieurs couleurs. Un jour, Clairwil propose une débauche singulière. C'est l'époque du carême, elle désire faire sa dévotion aux Carmes, dont le confesseur est un charmant religieux. Son projet est de se rendre à confesse, de lui échauffer la tête et de l'épuiser; puis de retourner à l'église, de communier, de recueillir les hosties et de « faire des horreurs sur ce misérable symbole de l'infâme religion chrétienne » (p. 582). La proposition est si folle que Juliette ne manque pas de sermonner sa préceptrice: " Dès que nous ne croyons pas en Dieu [...], les profanations que tu désires ne sont plus que des enfantillages absolument inutiles » (ibid.). Encore une fois, la tutrice doit admettre que l'élève a raison, mais Clairwil trouve une nouvelle justification : quoique les profanations soient des actes contradictoires pour les athées, elles embrasent la tête et empêchent le retour à la vertu, puisqu'une fois profanés, les objets tenus comme sacrés ne peuvent plus l'être. Pour conclure sa défense, Clairwil relance la réprimande : "Te l'avouerai-je, d'ailleurs, je ne te crois pas encore très ferme sur toutes ces choses-là » (ibid.). La réponse de Juliette est digne de la fermeté que le témoignage de la veuve met en question :

\footnotetext{
Ah! Clairwil, quelle est ton erreur [...], je suis peut-être plus rassurée que toi; mon athéisme est à son comble. N'imagine donc pas que j'ai besoin des enfantillages que tu me proposes pour m'y affermir; je les exécuterai puisqu'ils te plaisent, mais comme de simples amusements, et jamais comme une chose nécessaire, soit à fortifier ma façon de penser, soit à en convaincre les autres. (Ibid.)
}

La rhétorique de la jeune libertine convainc partiellement l'ancienne. Bien que Clairwil se dise sûre de l'étudiante, elle ne veut pas échouer dans

21. Michel Delon, « Notes de l'Histoire de Juliette », op. cit., p. 1463. 
le débat. Il faut poser l'argument à nouveau : l'hostie et le crucifix appartiennent à l'imaginaire chrétien vénéré en Europe; en les profanant, elle attaque l'opinion publique et anéantit les préjugés de son enfance. Elle ne vise pas Dieu précisément, " mais la religion, en tant que croyance humaine ${ }^{22}$ ». Ce " caractère violemment blasphématoire ${ }^{23}$ » serait une spécificité de Sade, selon Jean-Baptiste Jeangène Vilmer, qui différencie l'athéisme tranquille ou parfois passionnel des matérialistes de celui du marquis, beaucoup plus violent. Certes, le blasphème était une pratique courante à l'époque. Un délit durement puni représente toujours une transgression irrésistible. Puis le blasphème provoque, scandalise et enflamme la tête : c'est une arme qui attaque moralement par le langage, comme le fait physiquement le fouet. Mais il faut convenir qu'il s'agit d'un procédé contradictoire avec l'athéisme, puisque toute insulte présuppose l'existence et l'importance de l'être insulté. Si les héros de Sade nient et l'existence et l'importance de Dieu, leurs profanations ne sont que des fantaisies. «Soit, nous ne les ferons que comme un plaisir : [...] livrons-nous à cette plaisanterie par libertinage » (p. 582), répond la veuve à Juliette et répondrait peut-être aussi Sade, car enfin le paradoxe ne diminue en rien la jouissance de l'opération, bien au contraire.

\section{Le putanisme fanatique}

Le père Claude - le religieux des Carmes convoité par Clairwil - est vite séduit. Sous le prétexte d'apaiser l'embrasement des deux scélérates, il se montre aussi libertin qu'elles. L'orgie en petit comité se révèle extraordinaire : le coquin a " trois couilles » et un membre semblable à celui d'un «mulet » (p. 585). Afin de continuer la partie en plus grand nombre, la veuve lui demande s'il existe d'autres débauchés dans le couvent. La réponse affirmative entraîne des arrangements : le festin est fixé le jour de Pâques. Claude cependant, ne jouira pas de la bacchanale sacrilège. Quelques jours plus tard, Clairwil l'invite à la campagne, et plus personne ne le revoit : la tribade s'est fait composer le plus singulier godemiché avec le membre du malheureux. Plus que féministe, Clairwil serait l'image inversée du mâle prédateur et misogyne. Par la négation du sexe masculin et sa réduction à un outil de libertinage, elle prétend transgresser le rôle traditionnel de l'homme, sans le transformer socialement.

À la veille de Pâques, les deux scélérates se rendent aux Carmes. Le supérieur demande des nouvelles de Claude, elles lui assurent qu'elles ignorent son sort. Le sujet est oublié, les combattants arrivent : soixantequatre moines et dix novices. L'orgie dure toute la nuit et toute la journée de Pâques. Après le souper, Clairwil décide de se coucher sur le maître-autel

22. Jean-Baptiste Jeangène Vilmer, La Religion de Sade, Paris, Les Éditions de l'Atelier - Éditions Ouvrières, 2008, p. 45.

23. Ibid., p. 42. 
et d'y « foutre toute la nuit» (p. 620). Juliette crie : «Ah! Je ne peux plus foutre! » (p. 621). L'enseignante la dispense des orgies, mais se vante de sa propre résistance à l'excès par rapport à la délicatesse de l'apprentie : «[...] les flots de sperme qui m’ont inondé le cul et le con, n’ont fait que m'enflammer » (ibid.). Étourdie par l'extase de la longue débauche, la préceptrice est prise par un « mouvement d'effervescence, prononcé de l'air et du ton d'une énergumène » (ibid.). Tandis que l'impétuosité de l'écolière est équilibrée par la raison, celle de l'institutrice échappe complètement aux ordres de sa tête. L'heureuse apathie du discours devient dans la pratique une ode fanatique au putanisme. La femme qui se targue de maîtriser le sang-froid, de venger son sexe et d'immoler le sexe opposé, incarne un égarement d'adoration phallique :

Le putanisme est la vertu des femmes; nous ne sommes créées que pour foutre $[\ldots]$ et puisque le jour n'est pur, à mes yeux, que depuis que je connais l'art de foutre, c'est de cette seule époque que j'ai reçu l'existence... Des vits, oui, sacredieu, des vits, voilà mes dieux, mes parents, mes amis, je ne respire que pour ce membre sublime; et quand il n'est ni dans mon con, ni dans mon cul, il se place si bien dans ma tête, qu'en me disséquant un jour on le trouvera dans ma cervelle. (Ibid.)

\section{Le fantasme d'un crime pur et perpétuel}

L'étonnant est qu'à l'occasion de l'assassinat du père biologique de Juliette, quelques jours avant l'épisode précité, Clairwil réprimandait déjà l'excès d'enthousiasme de la jeune femme en en parlant à Noirceuil et à Saint-Fond : « [...] je lui trouve toujours le même défaut; elle ne commet le crime que dans l'enthousiasme, il faut qu'elle bande; et l'on ne doit jamais s'y livrer que de sang-froid» (p. 604). La veuve soutient que le crime doit stimuler la volupté, pas le contraire : "C'est au flambeau du crime qu'il faut allumer celui de ses passions, tandis que ce n'est qu'à celui des passions que je la soupçonne d'allumer celui du crime» (ibid.). Pour Juliette, la formule est plutôt réciproque : «Que le flambeau du crime s'allume à celui de la lubricité; réunis l'une et l'autre de ces passions, et tu verras ce qu'on retire de toutes deux » (p. 777), enseigne-t-elle à Olympe Borghèse. En effet, l'écolière a une habileté naturelle pour harmoniser la sensibilité avec le crime. Chez elle, l'enthousiasme amplifie l'énergie du vice à travers l'imagination, alors que chez Clairwil, les règles du libertinage sont beaucoup plus rigides. Dans ses dissertations, le crime occupe une place nodale et la volupté devient un accessoire : l'excitation n'est ni nécessaire, ni désirée pour l'accomplissement du vice. Le mérite du libertin se traduit par le contrôle de soi et l'exercice froid de ses turpitudes, il se passe d'adjuvants : "Je veux qu'elle trouve dans le mal dénué de toute luxure, l'entière volupté qui existe pour elle dans la luxure; je veux qu'elle n'ait besoin d'aucun véhicule pour exercer le mal » (p. 604). 
Il s'agirait du même effort que dans la vertu kantienne ${ }^{24}$. Le crime est imposé par devoir et non plus par penchant naturel. On assiste donc à une inversion de la morale de Kant, qui soutient la bienfaisance comme effort de la volonté et non comme inclination sensible, c'est-à-dire comme une empathie pour le malheur d'autrui. Pour Clairwil, le crime « doit être pur, indépendant de tout intérêt personnel ${ }^{25} \gg$ et de toute influence naturelle. Il faut ainsi soumettre la « sexualité instinctive ${ }^{26}$ » aux forces de la raison, dépasser la nature et accéder à une énergie criminelle absolue. La théorie du crime pur s'appuie sur l'apathie pour configurer une véritable ascèse. Poussant cette métaphysique encore plus loin, la veuve aboutit à la transcendance du vice :

Je voudrais $[\ldots]$ trouver un crime dont l'effet perpétuel agît, même quand je n'agirais plus, en sorte qu'il n'y eût pas un seul instant de ma vie, où même en dormant, je ne fus cause d'un désordre quelconque, et que ce désordre pût s'étendre au point qu'il entraînât une corruption générale, ou un dérangement si formel, qu'au-delà même de ma vie, l'effet s'en prolongeât encore... (p. 650)

C'est le «même fantasme d'un libertinage au-delà de la mort, revendiqué déjà par Saint-Fond ${ }^{27}$ ». Les deux libertins emploient des artifices équivalents pour braver les limites de la nature et assurer la pérennité de leurs forfaits : tandis que le ministre compte sur l'action des molécules malfaisantes, la veuve cherche un moyen de prolonger ses atrocités à l'infini. Le parallèle entre les personnages est explicite : leur modèle de libertinage est tellement rigoureux qu'il ne s'applique pas sans excéder les paradoxes. Chez leurs complices, cette incohérence entre discours et pratiques provoque des sentiments ambigus. Saint-Fond est tué par son ami intime, Noirceuil. Clairwil subit le même destin. Malgré sa métaphysique sanguinaire, son dernier crime ne serait ni pur ni perpétuel. À Ancône, la sorcière Durand propose à l'enseignante d'empoisonner Juliette pour deux mille louis. Mais l'affection pour l'apprentie l'emporte sur le devoir du mal absolu. La préceptrice ne réussit pas à cacher son agitation et avoue à la chère écolière la dangereuse proposition. C'est inutile. Déjà séduite par la mégère, la jeune se méfie de la confession : au risque de devenir victime, elle préfère jouer le bourreau. Emportée par la nouvelle amie, Juliette tue l'ancienne flegmatiquement.

De ce point de vue, le dénouement chez Sade est plus tragique que chez Laclos : Merteuil est condamnée à une " mort mondaine », Clairwil subit la «mort physique ${ }^{28} »$. Ce qui est sûr, c'est que les deux grandes

24. Voir Michel Delon, L'Idée d'énergie, op. cit., p. 408-409.

25. Michel Delon, « Notes de l'Histoire de Juliette », op. cit., p. 1469.

26. Giorgio Cerruti, «Le paradoxe sur le comédien et le paradoxe sur le libertin, Diderot et Sade ", Revue des sciences humaines, $\mathrm{n}^{\circ}$ 146, 1972, p. 245.

27. Michel Delon, « Notes de l'Histoire de Juliette », op. cit., p. 1479.

28. Nous empruntons les deux expressions à Michel Delon, Le Savoir-Vivre libertin, op. cit., p. 295. 
architectes du libertinage féminin tombent également dans le malheur : la marquise, « jalouse comme une amante de tragédie ${ }^{29}$ », la veuve, empressée comme une héroïne sentimentale. Si chez Laclos la tragédie rétablit peutêtre la morale, chez Sade « toute prétention rationnelle ${ }^{30}$ » semble trouver ses limites, la philosophie ayant aussi ses dangers : « À force d'analyser la nature, nous ressemblons au chimiste qui se ruine pour faire un peu d'or. Élaguons, mais n'anéantissons pas tout, parce qu'il y a dans la nature des choses très singulières et que nous ne devinerons jamais ${ }^{31} \gg$. Le rapport entre Juliette et Durand - la Brinvilliers contemporaine - serait-il une de ces « choses très singulières » dont parle Sade ? Il suffit de rappeler que la protagoniste est la « femme unique en son genre » (p. 1261) et la sorcière, la maîtresse qui l'accompagne au sommet de son itinéraire. Tant l'une que l'autre savent que l'impératif de dominer tout mouvement naturel se trahit facilement. Ce secret a échappé à Clairwil. Son apathie et son féminisme se dissipent si vite qu'elle s'en aperçoit à peine. Trop fière de sa froide raison, elle ne se garde ni de l'enthousiasme, ni du fanatisme, ni du fantasme. Trop occupée à attaquer la conduite criminelle de Juliette, elle ne se défend pas de l'impétuosité fascinante de l'apprentie ${ }^{32}$. Le libertinage de l'institutrice, transitoire par nature, est surpassé par le libertinage de l'écolière, dont le mouvement ne peut être qu'ascendant.

Clara Carnicero de Castro Université de São Paulo ${ }^{33}$

29. Jean Fabre, «Interventions sur la communication de Jean Biou », dans Le Marquis de Sade, Paris, Armand Colin, 1968, p. 112.

30. Jean Fabre, «Préface aux Crimes de l'amour », dans Idées sur le roman : de Madame de Lafayette au marquis de Sade, Paris, Klincksieck, 1979, p. 207.

31. Note de Sade, «Faxelange, ou Les torts de l'ambition », dans Les Crimes de l'amour : nouvelles hérö̈ques et tragiques, éd. Michel Delon, Paris, Gallimard, 1987, p. 64.

32. Voir Annie Le Brun, Soudain un bloc d'abîme, Sade, Paris, Gallimard, 1989, p. 305-307. 33. Ce travail de recherche a été en partie financé par la Fundação de Amparo à Pesquisa do Estado de São Paulo (FAPESP) dans le cadre de mon post-doctorat à l'Université ParisSorbonne. Que l'agence et l'université en soit remerciées. 